\title{
A CLINICO-PATHOLOGICAL STUDY OF ATELECTASIS IN PULMONARY TUBERCULOSIS
}

\author{
BY \\ LESLIE J. TEMPLE \\ From the Department of Surgery, University of Liverpool
}

(RECEIVED FOR PUBLICATION APRIL 12, 1955)

The literature of atelectasis in pulmonary tuberculosis is both vast and controversial. In the past, attention has been drawn mainly to atelectasis of lung complicating artificial pneumothorax. With the decrease in this method of treatment, however, the problem of atelectasis has not disappeared. The association between bronchial obstruction and atelectasis was noted by both Gairdner (1851) and by Bastian (1871), but the examples they observed were not tuberculous. The mechanism of this type of atelectasis has been very fully discussed by Pasteur $(1908,1914)$, by Lee Lander (1936, 1946), and by Lee Lander and Maurice Davidson (1938a and b). None of these observations, however, can be taken as necessarily applicable to atelectasis in pulmonary tuberculosis.

Coryllos (1933) examined the question exhaustively. He considered that atelectasis was always due to bronchial obstruction and believed that obstruction of the bronchus with atelectasis invariably produced cavity closure unless the cavity wall was too rigid. That his concept is not entirely correct is shown in the first illustration he gives of atelectasis of the right upper lobe ; after the induction of artificial pneumothorax the "atelectatic" upper lobe is clearly larger than the aerating lower lobe. The condition, therefore, is not one of true atelectasis. Moreover, his illustrations of stem cavities show a patent bronchus opening into the cavity and surrounding it is a ring of atelectasis. An explanation of this condition is offered in the ensuing section. Coryllos denied the existence of pressure atelectasis and considered that closure of cavities was always due to bronchial obstruction.

Erwin (1939) reviewed a similar group of cases and he too believed that atelectasis was always due to bronchial obstruction but noted its rarity as a segmental disease. Pinner (1945) believed that uncomplicated atelectasis in pulmonary tuberculosis was rare and he had never in fact seen it at necropsy. He pointed out the snares in the diagnosis of atelectasis and observes that many examples so diagnosed are not the simple atelectasis which one would expect if the collapse were due to bronchial occlusion. More recently Clegg (1953), in discussing the distal lymphatic endobronchial spread of tuberculosis, has shown in the later stage of this lesion that when the bronchial wall has been destroyed patches of atelectasis appear round the bronchus. If this happens in relation to several small bronchi these patches tend to approximate and finally to fuse. However, in those examples of healed disease of this type in which he shows a segmental bronchus completely occluded, there is no atelectasis. This suggests that his explanation is inadequate, and that atelectasis cannot be due merely to the blocking of a small peripheral bronchus. Whitwell (1952) in writing of bronchiectasis has shown that even though all communications between a segmental bronchus and the lung may be sealed off, the alveoli remain well aerated-evidently from other bronchi.

Other theories of the causation of atelectasis have been put forward, and the suggestion that reflex spasm of the involuntary muscle fibre of the lung is responsible for it has been made by Xalabarder (1949) and Hoffstaedt (1953). They recognize that bronchial obstruction alone is not always responsible for the atelectasis of part of a lung, but suggest that it may be one of the stimuli that result in "reflex atelectasis."

The clinical aspects of this condition have been discussed by Sadler (1954), who followed up patients who developed atelectasis after treatment by artificial pneumothorax at the Cheshire Joint Sanatorium. He tried to trace the subsequent course of the disease in patients with and without atelectasis. He demonstrated a clear correlation between atelectasis and the onset of pleural effusion, although he believes this to be due to the greater extent of the disease in the patients with atelectasis. The long-term follow-up is limited to 
159 patients out of 266 , and only in three cases out of 20 of persistent cavitation was a follow-up obtained. This in itself is an indication of the bad prognosis associated with atelectasis in cavitating disease. He concludes like Erwin that permanent atelectasis is a desirable end-result for a severely diseased lobe. Nevertheless in the final table, after excluding patients with a persistent cavity, he shows that within eight years the death rate for total atelectasis is $15.4 \%$, for lobar atelectasis $6.8 \%$, for segmental atelectasis $5.9 \%$, and for non-atelectatic disease $2.3 \%$.

Houghton (1950) stated: "Atelectasis as a complication of pneumothorax is a constant precursor of tuberculous empyema and is frequently associated with ultimate relapse." Many chest physicians with whom the present author has worked would, from their own experience, echo this statement. Maher-Loughnan (1950) also showed that patients with atelectasis complicating an artificial pneumothorax are very likely to develop pleural effusion, empyema, and bronchial spread. Not all writers, however, accept this, and Farquharson (1951) does not think that the prognosis in such patients is necessarily bad.

Coello (1951) and Coello and Nagley (1948), observing atelectatic areas through the thoracoscope, tried to distinguish two types of atelectasis -one relatively benign, the other dangerous and liable to lead to empyema. Similar conclusions are reached in the present paper, and a pathological basis suggested.

\section{Method of InVESTigation}

An examination of specimens of lung or portions of lungs resected for pulmonary tuberculosis has been carried out since 1949. Each specimen is distended and fixed with formalin at such a pressure as to maintain it in its original life size. It is then embedded in gelatin, frozen, and sliced as a whole into sections $500 \mu$ thick in a coronal plane, sectioning as far as possible the whole specimen. At appropriate points through the blocks small pieces of tissue are removed for microscopic examination. The methods used throughout have been a modification of those of Gough and Wentworth (1949). An examination is carried out of every section so that it is possible to build up the entire anatomy of the specimen. The bronchi are followed through the sections, and their relationship to cavities and other lesions is noted. A few representative sections are mounted. To this gross anatomical study is added a report of the histology, and then an attempt is made to correlate the picture presented with the radiographs and the clinical details of the patient before and after operation. In this way 125 specimens have so far been examined, and a preliminary attempt has been made to separate them into different clinical types of disease. This paper concerns those patients on whom a pre-operative diagnosis of atelectasis was made.

The resections in every one of these patients were carried out because clinically it was considered advisable to do so, and not because of any preconceived theory that resection should be done in every patient in whose lungs a patch of atelectasis was seen. To this extent only are the patients a selected group and it was inevitable that the selection was largely of those whose clinical course was not satisfactory.

\section{ILLUSTRATIVE RECORDS}

In the first group of patients, there was complete or almost complete stricture of a main bronchus. Most of the specimens in this group are whole lungs with stricture of a main bronchus, but there are a few in which a lobar bronchus only is involved, the interlobar fissure is complete, and atelectasis is limited to a lobe. All these specimens show gross bronchiectasis, and the alveolar tissue consists of an atelectatic rind around dilated bronchi filled with inspissated tuberculous pus. Fig. 1 is a section through a complete right lung. In this patient the right main bronchus was only $2 \mathrm{~mm}$. in diameter at the carina, and radiographic examination showed that the right lung was non-aerating. The sputum occasionally contained tubercle bacilli and on two occasions a minor spread of disease to the left lung took place. On first sectioning the resected lung, all the spaces were filled with caseous material (some of it has dropped out in the mounted section). Microscopy showed the caseous masses to be contained by a layer of ciliated epithelium, that is, they are lying in greatly distended bronchi. The lung tissue remaining between the bronchi is fibrotic and the small foci in it well healed. Fig. 2 is a similar specimen which actually demonstrates the stricture. All the caseous contents have dropped out. Fig. 3 shows a left lung with atelectasis of the upper lobe only. The main bronchus was narrowed, but the upper lobe bronchus had disappeared completely. The upper lobe consists of dilated bronchi embedded in atelectatic fibrotic lung.

All these specimens show pathological features identical with a group of lungs diagnosed pre- 


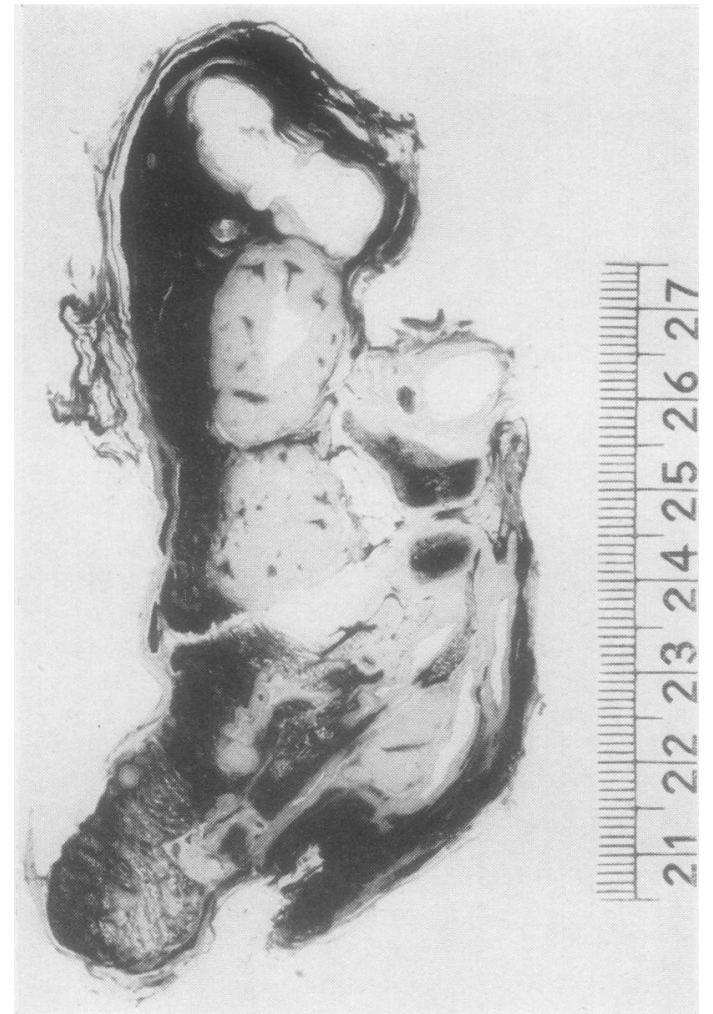

FIG. 1

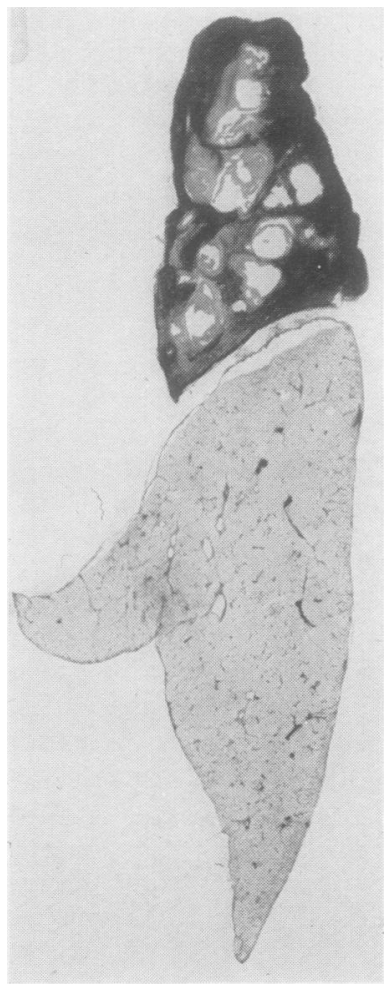

FIG. 3

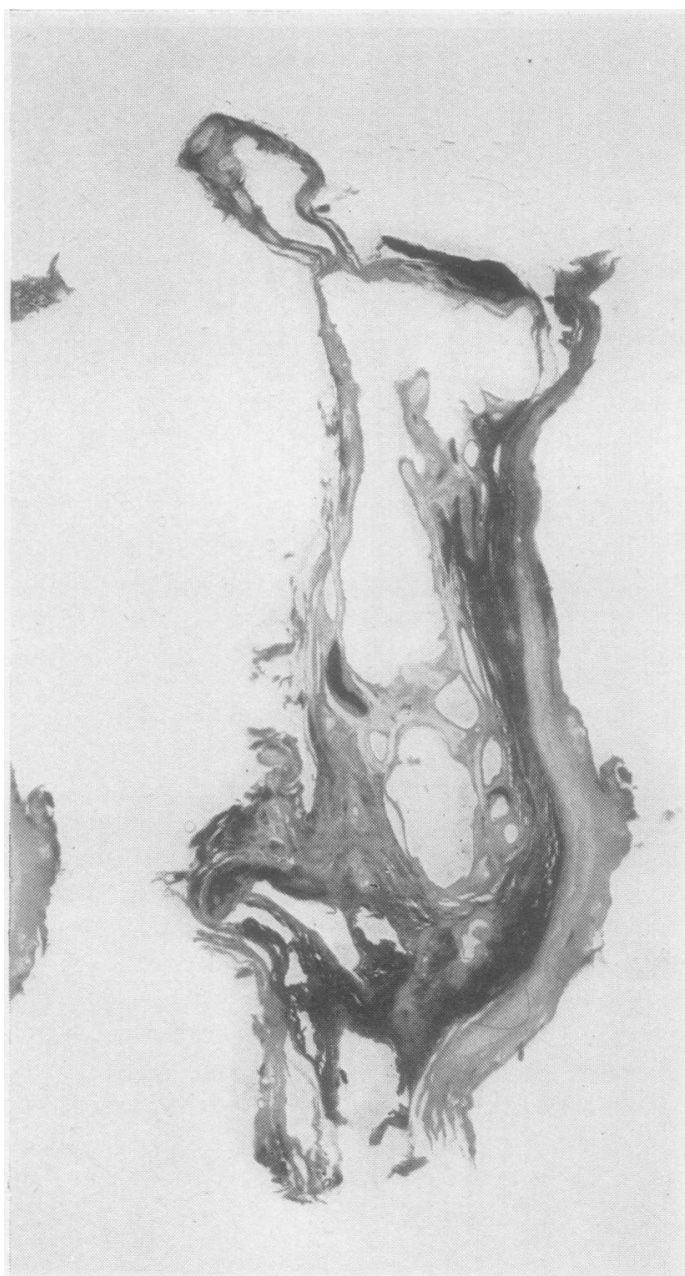

FIG. 2

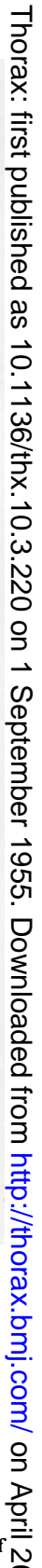

FIG. 1.-Section of a totally atelectatic lung with bronchi full of
caseous material. FIG. 2.-Section of whole lung showing bronchial stricture.

FIG. 3.-Lung with stricture of upper lobe only. 
operatively as tuberculous bronchiectasis or destroyed lung. They differ only in the presence of a major bronchial stricture. In the group without a stricture the bronchi are largely empty and the pre-operative radiological appearance has suggested extensive bronchiectasis or cavitation and not atelectasis. In both types there is little evidence of active disease and the main danger to the patient appears to be due to the retention of caseous material or pus which might on occasion flood the healthy lung.

The second group of patients showed atelectatic lobes which contained large, patent cavities. The alveolar tissue forms the thick wall of the cavity. Fig. 4 is the radiograph of a young woman who had a cavity in the left upper lobe. An artificial pneumothorax produced atelectasis of the lobe which persisted even after the artificial pneumothorax was abandoned. In spite of total collapse of the lobe the cavity remained patent. Fig. 5 is a section of the removed lobe. The cavity is lined by caseous material and granulation tissue which extends irregularly into the surrounding atelectatic lung. There is almost no fibrosis and little localization of the disease. The cavity did not collapse with the lobe for the same reason that the cavities in a Gruyere cheese do not collapse-the caseous wall is too rigid. The lobar bronchus shows neither narrowing nor marked endobronchial disease.

The third group of patients were those showing only partial atelectasis of a lobe. Most of these were removed from under an artificial pneumothorax. It was in this group that a number of spəcimens diagnosed pre-operatively as atelectasis proved to have no collapse of lung, but a solid mass of disease. These were naturally rejected from the series. In none of these specimens of partial atelectasis did the area collapsed correspond to a segment and in no instance was stricture of a segmental bronchus found.

Fig. 6 is the tomograph of a patient whose pulmonary cavity closed while in a sanatorium and whose treatment included an artificial pneumothorax. As is shown in the radiograph a patch of atelectasis persisted in the left upper lobe. The clinician in charge was unhappy about this patient, not because of the radiological appearance, but because the clinical progress was unsatisfactory. The lobe was therefore resected. Fig. 7 is a section through the lobe; it shows two caseous nodules lying astride an intersegmental plane and surrounded by a mass of atelectatic lung. A section more anteriorly (Fig. 8) shows fusion of nodules and disease spreading into the atelectatic area. A photomicrograph (Fig. 9) of the caseous edge of

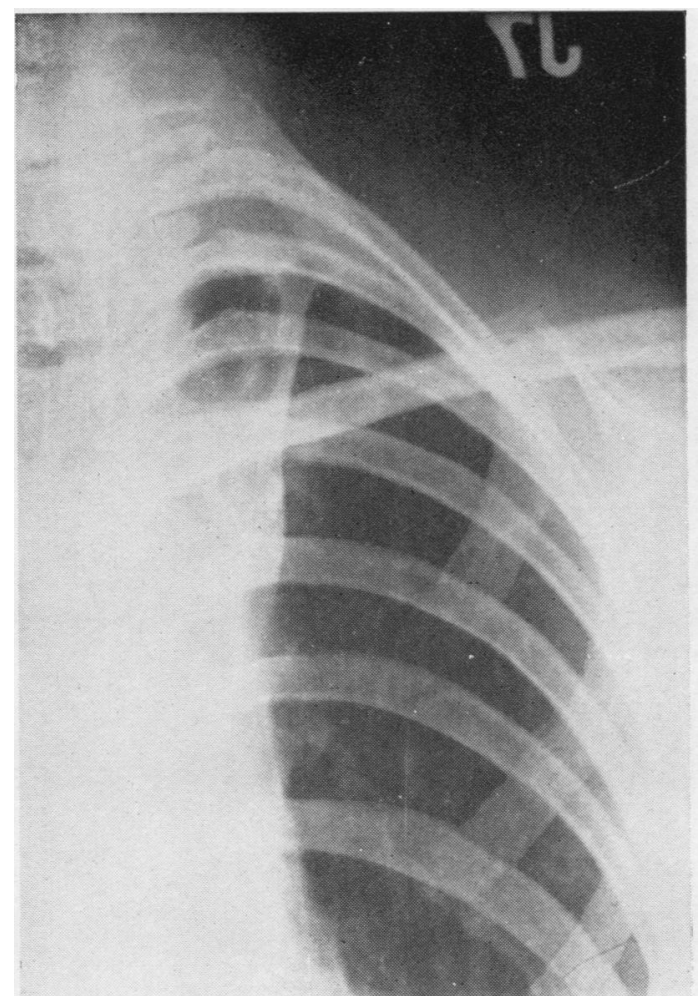

FIG. 4.-Radiograph showing atelectatic left upper lobe with cavity.

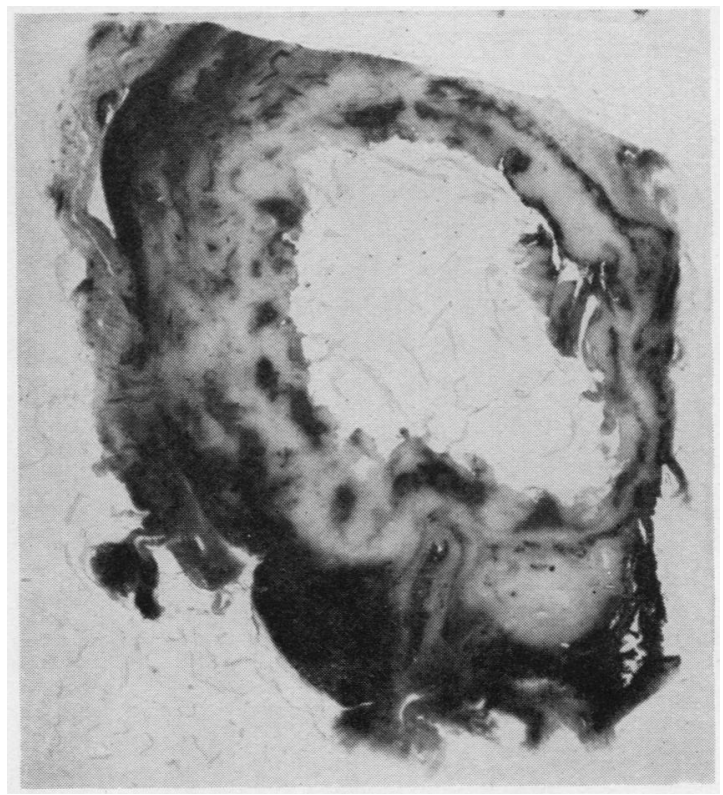

FIG. 5.-Section of lobe removed from patient whose radiograph is shown in Fig. 4. 


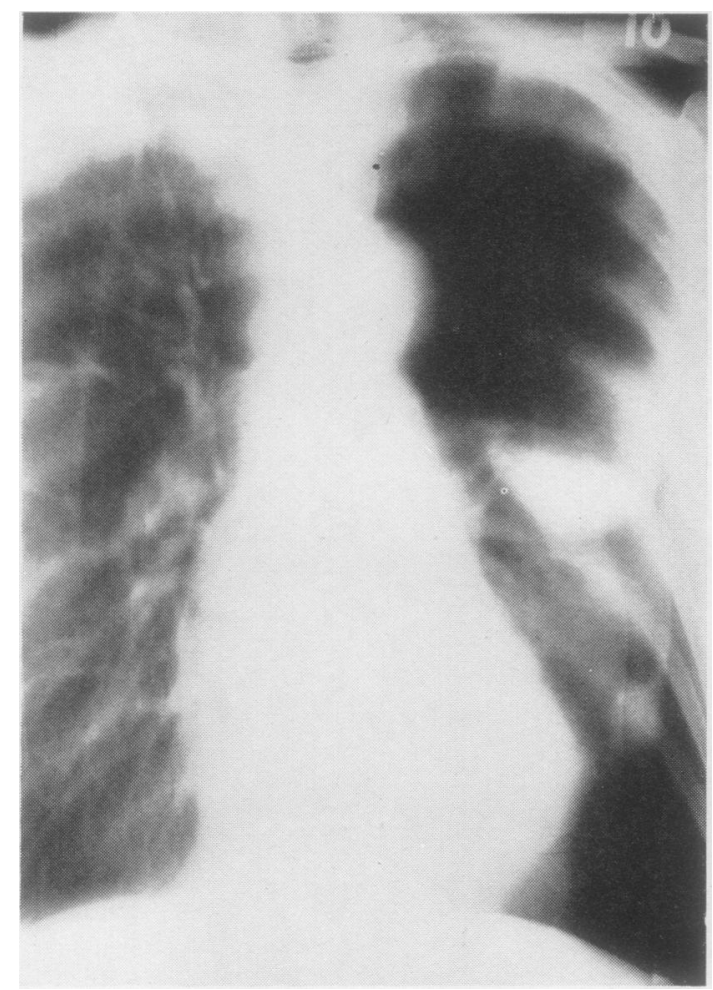

FIG. 6.-Tomogram showing area of atelectasis in left lung.

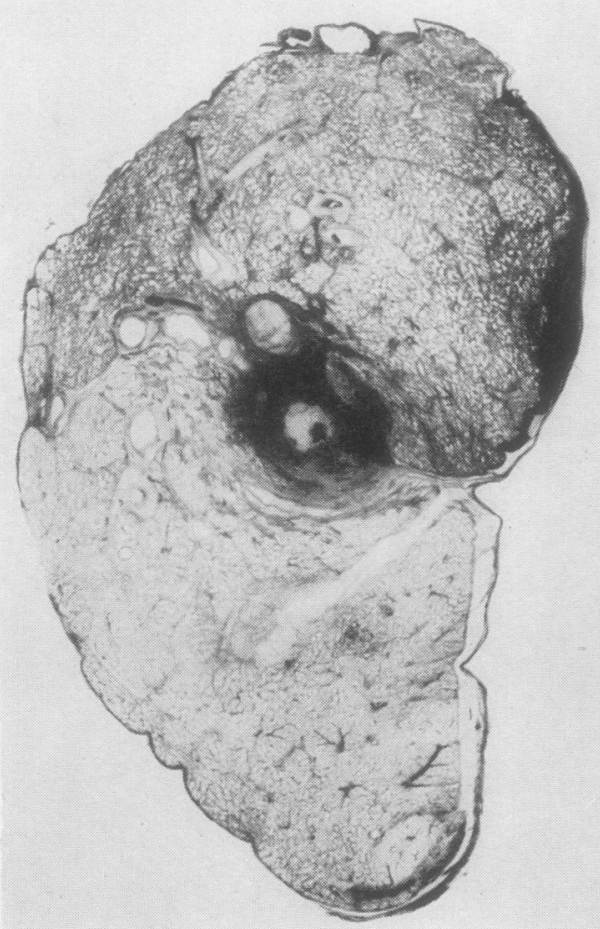

Fig. 7.-Section through upper lobe from Fig. 6.

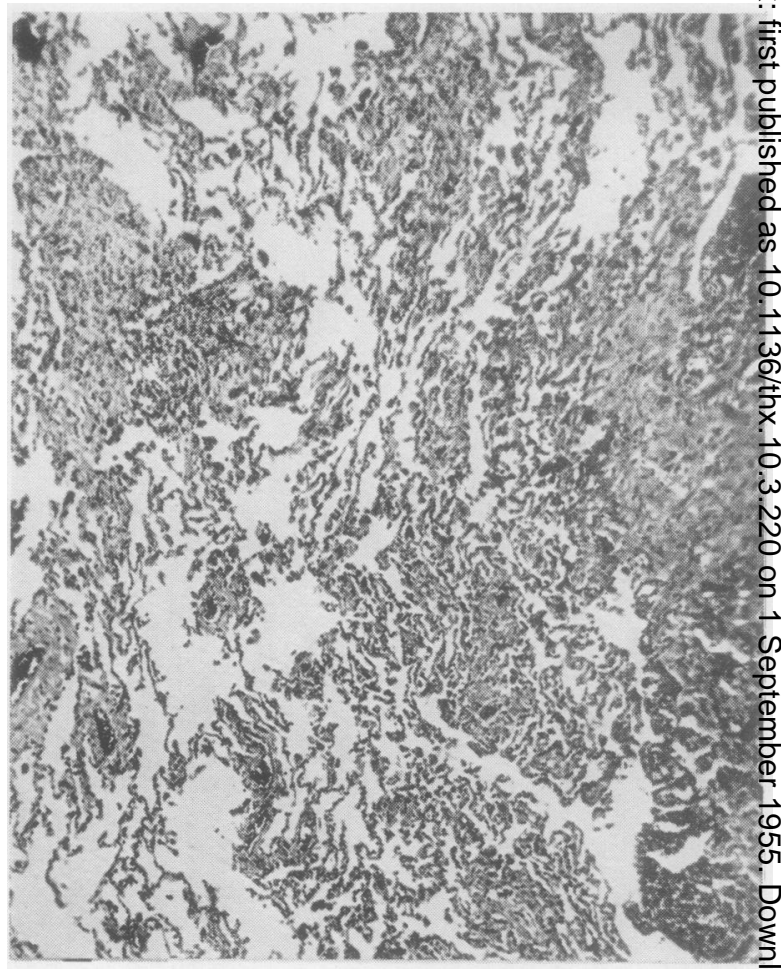

FIG. 9.-Photomicrograph from edge of tuberculous area in the same case.

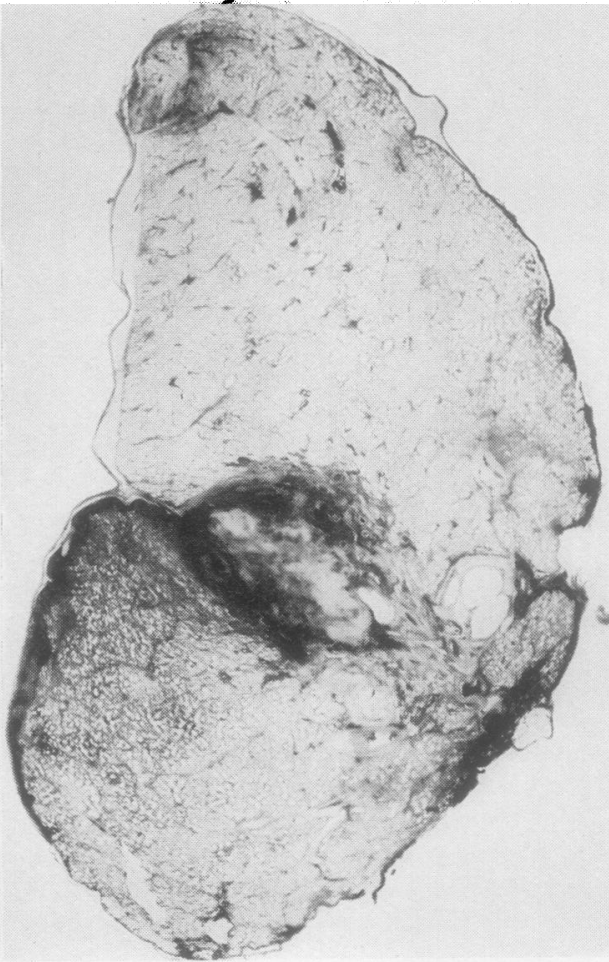

Fig. 8.-Section through same lobe further forwards. 
the disease makes it clear that there is no delimiting encapsulation. The tuberculous process is extending freely into the surrounding compressed and flattened alveoli. The absence of any fibrosis underlines what might be called the malignancy of the advancing tuberculous disease.

Fig. 10 is a section of a lobe showing patchy atelectasis between solid nodules of disease ; localization of the lesion is poor and incomplete. This specimen was removed from a patient whose radiograph showed areas of atelectasis after induction of an artificial pneumothorax.

Fig. 11 is the radiograph of a patient with an artificial pneumothorax on the left side. Partial atelectasis of the upper lobe is present. Section of this lobe (Fig. 12) shows two areas which consist of caseous nodules embedded in atelectatic lung. The histological examination of these areas revealed actively spreading disease.

In the patient whose lung section is illustrated in Fig. 13 half of the upper lobe is atelectatic, lying compressed between irregularly fusing nodules of disease. The area corresponds to no segmental distribution.

In another patient, who developed an area of atelectasis under an artificial pneumothorax, the

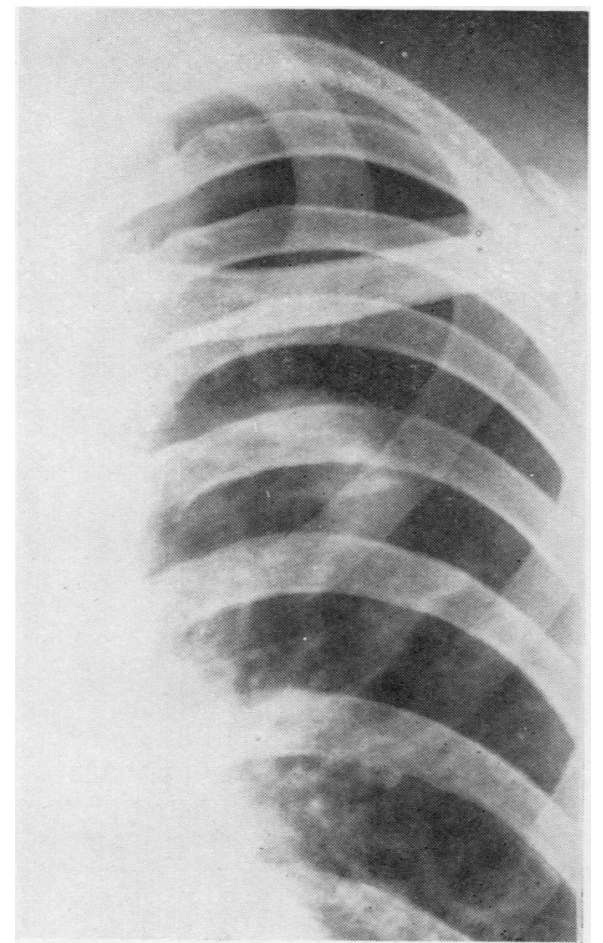

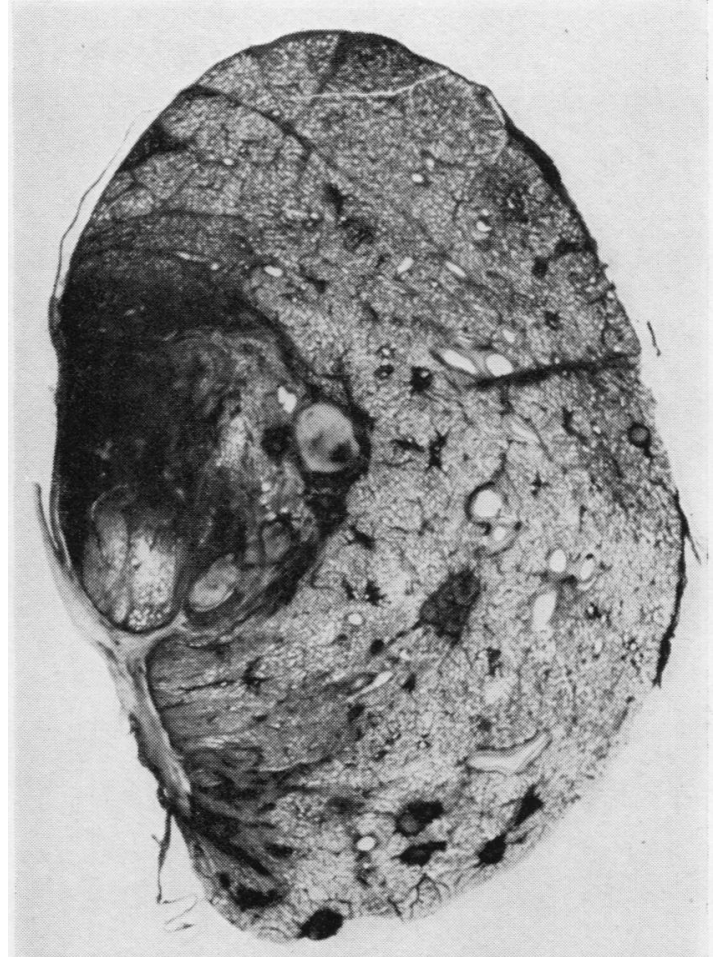

Fig. 10.-Tuberculous nodules and surrounding atelectasis.

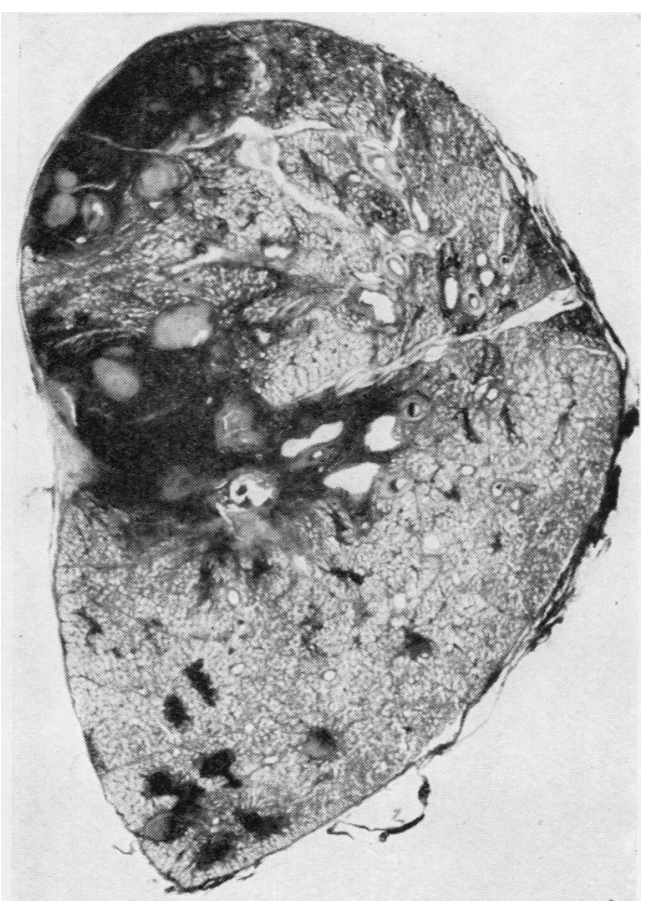

FIG. 11.-Radiograph showing partial atelectasis of right upper lobe $F[3$. 12.-Section through lobe removed from the same patient. under an artificial pneumothorax. 


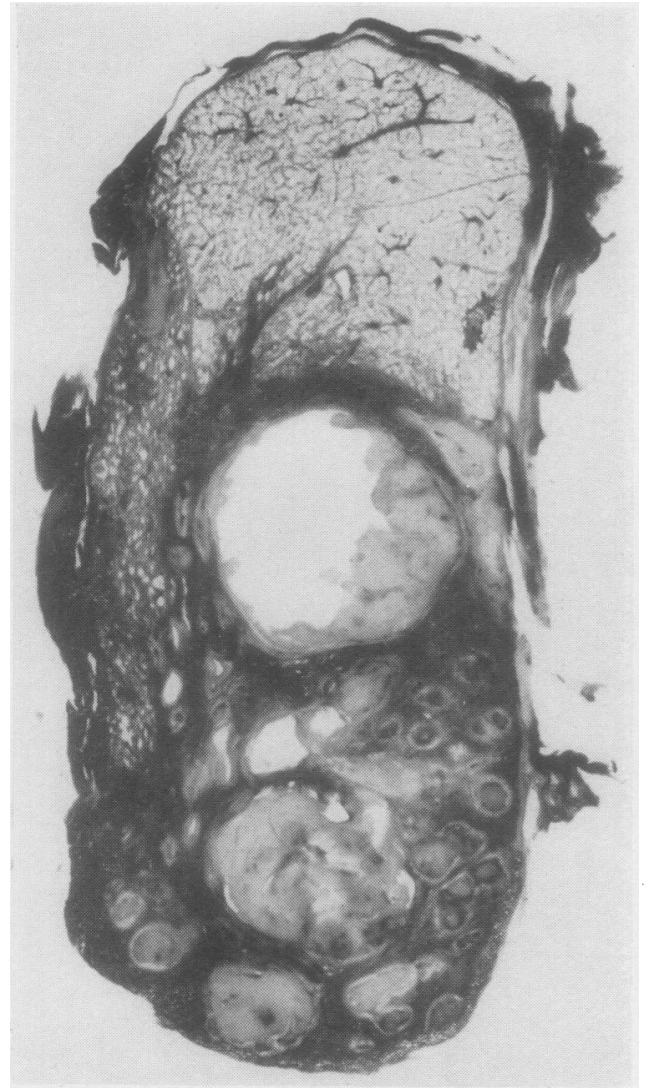

FIG. 13.-Caseous nodules and compressed surrounding lung.

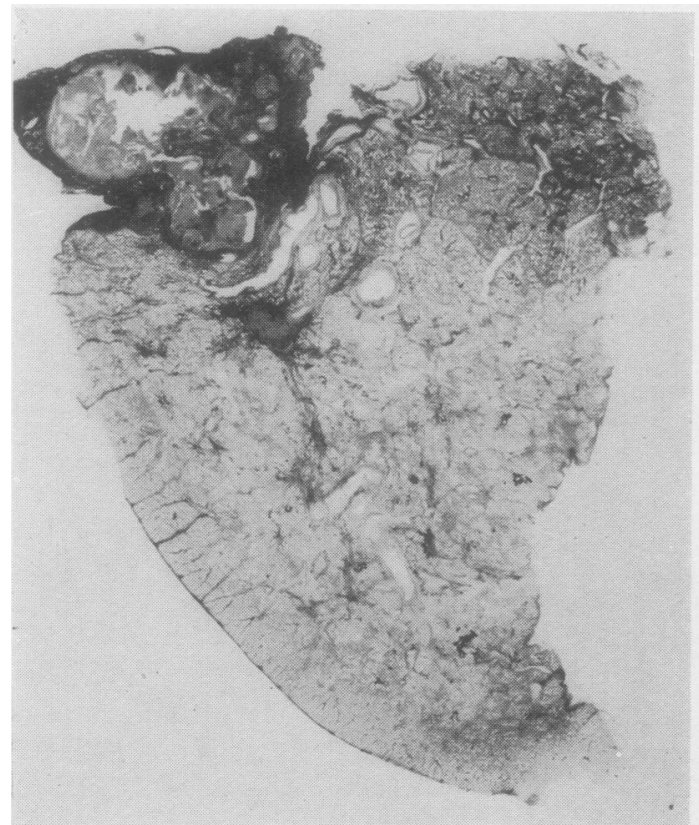

FIG. 15.-Caseous lesion with atelectasis in apical segment of lower lobe and similar lesion below the apical bronchus.

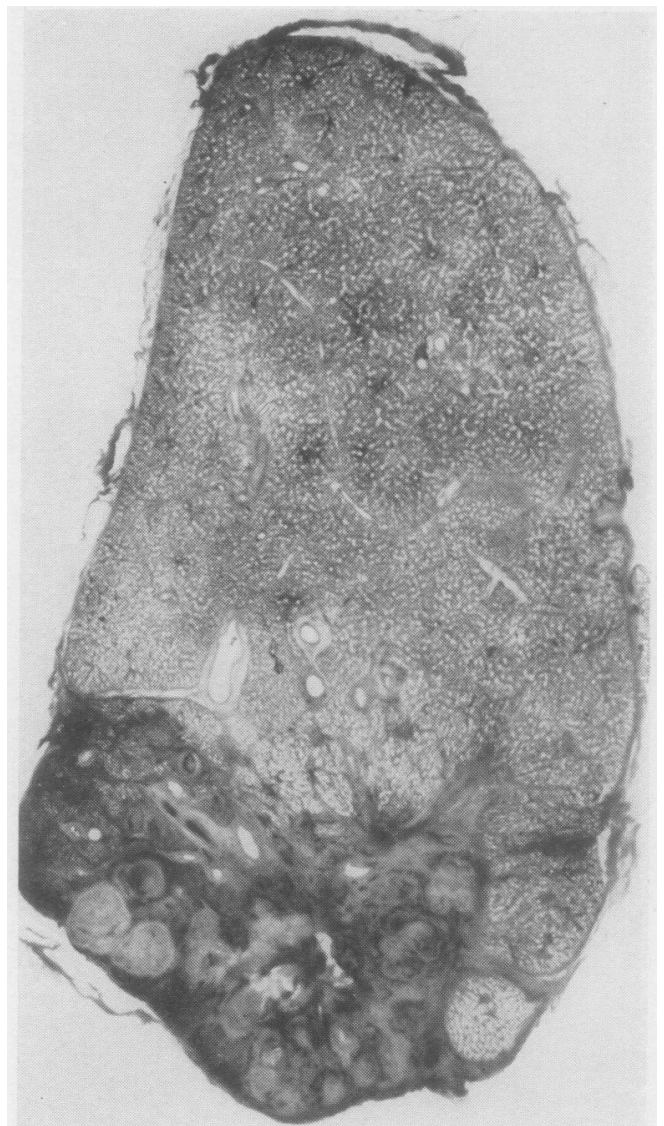

Fig. 14.-Caseous nodules and surrounding compressed lung.

artificial pneumothorax was nevertheless maintained, and eventually an effusion formed.으 Lobectomy was later carried out, and the specimen (Fig. 14) shows the airless alveoli compressed be-io tween nodules of actively spreading disease.

The next example illustrated (Fig. 15) had a pneumoperitoneum and phrenic crush before 3 removal of the left lower lobe. The apical caseous 을 mass has a rind of atelectatic lung around it and a similar small nodule near by-not in the apical 을 segment - has a similar rind. There was no gross disease of the segmental bronchi.

\section{Discussion}

All the specimens examined, including the $\omega$ examples described, seem to fall into one or other of two different pathological entities. On the one hand there are the destroyed lungs with bronchial $\stackrel{\oplus}{\oplus}$ stricture. What remains of the lung tissue is ? fibrotic and completely airless; it shows excellent $\frac{0}{0}$ healing, and these lungs are dangerous only when $\vec{D}$

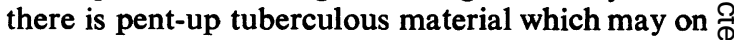
occasion flood over and infect the opposite lung. 음 In these patients the atelectasis is not in itself harmful. 
The other pathological entity is shown in the examples described of lobar atelectasis and partial atelectasis of the lobe. This type of disease is characterized by the relentless spread of active infection into the surrounding atelectatic lung with histological evidence of inadequate resistance. In the past, partial atelectasis in a lobe has been regarded as segmental, and associated with obstruction of the corresponding bronchi. In fact, in no case examined was a segmental bronchus found to be involved by the disease, and the distribution of the lesions followed no segmental pattern. This could have been anticipated from the experience of all thoracic surgeons that a segmental bronchus can be clamped at operation without producing any atelectasis. When a lung is relaxed, as, for example by artificial pneumothorax, the structures in it tend to get smaller; tuberculous lesions, such as cavities and fibrotic infiltrations, will presumably contract in proportion to or even selectively to a relatively greater extent than the surrounding alveoli. If, on the other hand, the disease is solid or infiltrating into the surrounding tissue so that the alveoli contain oedema fluid and their walls show cellular infiltration, the diseased portion of lung will be rigid and cannot occupy a lesser volume when the whole lung volume is reduced. Consequently the surrounding alveoli are compressed by this islet of solid material in the collapsing lung and atelectasis forms in a layer around the lesion. Where a number of lesions are in fairly close proximity collapse will bring them closer together and their rinds of atelectatic lung are likely to fuse into a single mass which becomes visible radiologically as a partial atelectasis. This has been clearly demonstrated by Clegg in the work previously referred to. Fig. 16 is an attempt to illustrate this phenomenon diagrammatically. Here, atelectasis is a manifestation that the disease is still actively infiltrating into surrounding lung.

A similar argument applies with regard to cavitating disease. If the cavity wall is thin and fibrotic it will at least diminish in proportion to the relaxation of the lung (Fig. 17). On the other hand, if the disease is still actively infiltrating into and destroying alveolar tissue, the wall is rigid with caseous material and oedema, and it cannot relax. It is against this rigid lesion that the surrounding alveoli are compressed and become airless. (Fig. 18, which represents this diagrammatically, should be compared with Fig. 5.)

\section{Conclusions and Summary}

A clinico-pathological investigation of atelectasis in pulmonary tuberculosis indicates that it
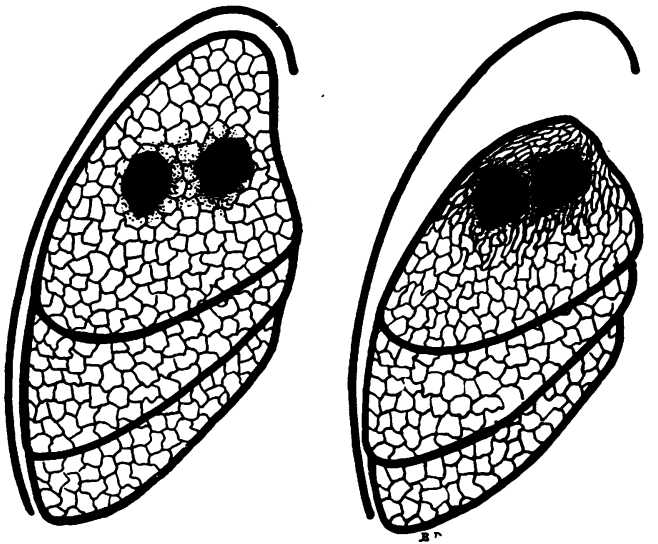

FIG. 16.-Diagram illustrating the effect of artificial pneumothorax on two active lesions with compression of intervening lung.
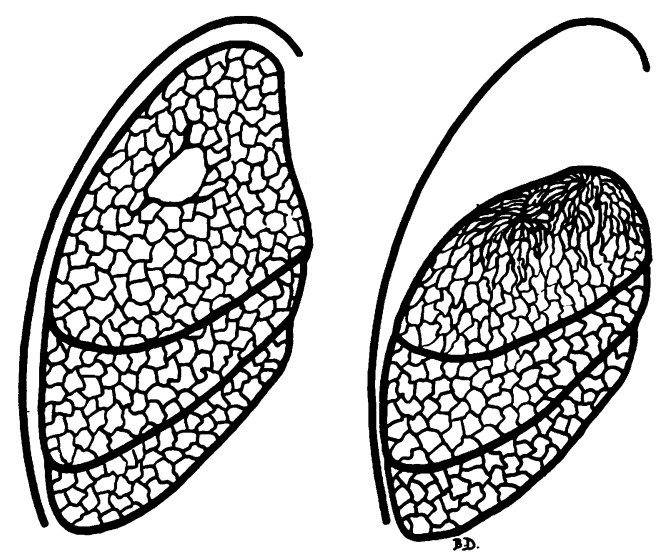

FIG. 17.-Fibrotic healing cavity closing with artificial pneumothorax .
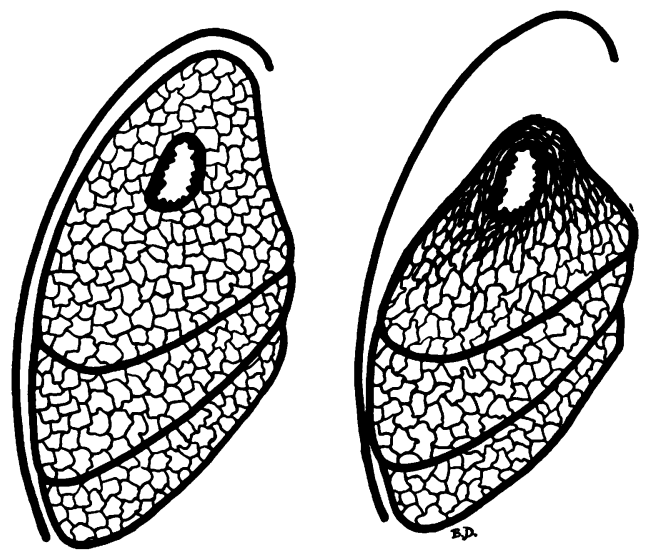

Fig. 18.-Thick-walled cavity failing to close with artificial pneumothorax and with compression of surrounding lung. 
may be comparatively harmless or it may be dangerous. Collapse of a whole lobe or of a lung may be due to bronchial occlusion, either by reason of tuberculous stricture, or of obstruction by tuberculous glands from without or accumulated secretions within the bronchus. The condition which results is essentially one of bronchiectasis with or without the retention of caseous material in the dilated bronchi. Coello's "safe atelectasis" is probably of this nature.

Moreover, a lung with fairly extensive tuberculosis may, after collapse therapy, show condensation of the disease, but no true compression of alveoli; the patient does well, and experience shows that this is in fact a healing stage in the disease.

On the other hand, if the lesion in the lung is comparatively small, yet after the induction of artificial pneumothorax or other measures of collapse appreciable areas of atelectasis appear, then the disease should be regarded as highly active. Obstruction of the segmental bronchi does not appear to be a causal factor. The alveoli surrounding the lesion are compressed by the rigid mass of active tuberculous infiltration and oedema. This type of "compression atelectasis" is a danger signal to warn that the disease is active and still advancing and that it may sooner or later involve the pleura, with consequent tuberculous empyema. Furthermore, it is not surprising that cavities with similarly infiltrated walls fail to close when collapse therapy is attempted.
While I take sole responsibility for all the views expressed, I must acknowledge my indebtedness to $\frac{}{\sigma}$ my various colleagues on the Liverpool Chest Unit $\frac{\bar{c}}{\omega}$ both for letting me have some of the specimens and $\vec{\Phi}$ for criticism and discussion. In particular I should like to thank Dr. Robert Coope. All the technical on work of this investigation has been carried out by $\vec{O}$ Mr. Harold Nevin and Miss Maureen Litchfield, of the Liverpool Department of Surgery. I have is $\vec{\omega}$ thank Professor Gough, of Cardiff, for the patient 0 instruction so kindly given me in his laboratory tech- $\overrightarrow{2}$ niques. The work was carried out at the Department of Surgery, University of Liverpool, with the help and $\overrightarrow{0}$ encouragement of Professor Charles Wells and with $\omega$ the aid of a grant from the United Liverpool $N$ Hospitals.

\section{REFERENCES}

Bastian, H. C. (1871). In A System of Medicine, ed. J. R. Reynolds. Macmillan, London. Vol. 3, p. 830

Clegg, J. W. (1953). Thorax, 8, 167.

Coello, A. J. (1951). J. thorac. Surg., 21, 135

- and Nagley, M. M. (1948). Tubercle, 29, 231

Coryllos, P. N. (1933). Amer. Rev. Tuberc., 28, 1

Erwin G. S. (1939). Brompton Hosp. Rep., 8, 43

Erwing. Su (1951). Tubercle 32, 08.

Farquharson, M. (1951). Tubercle, 32, 108.

med. Sci., 12, 440.

(1949), J. Fac. C Radiol., Lond., 1, 28

Hoffstaedt, E. G. W. (1953). Tubercle, 34, 234

Houghton, L. E. (1950). Ibid., 31, 50.

Lander, F. Lee (1936). Proc. roy. Soc. Med., 29, 1383.

- (1946). Thorax, 1, 198.

(19) Brit med J., 1, 1047.

(1938b), Brit J Radiol., 11, 65.

Maher-Loughnan, G. P. (1950). Tubercle, 31, 74

Pasteur. W. (1908). Lancet, 2, 1351

Pasteur. W. (1908). Lancet, 2, 1351

Pinn (1914). Brit. J. Surg., 1, S87. Springfield Illinois

Sadler, R. L. (1954). Brit. med. J., 1, 359.

Whitwell, F. (1952). Thorax, 7, 213

Xalabarder, C. (1949). Tubercle, 30, 266. 